PROCEEDINGS OF THE

AMERICAN MATHEMATICAL SOCIETY

Volume 126, Number 12, December 1998, Pages 3619-3623

S 0002-9939(98)04489-X

\title{
BOREL AUTOMORPHISMS WITH NO FINITE INVARIANT MEASURE
}

\author{
S. EIGEN, A. HAJIAN, AND B. WEISS
}

(Communicated by Mary Rees)

\begin{abstract}
An uncountable family of non-isomorphic Borel automorphisms which do not preserve any finite measure is presented.
\end{abstract}

\section{INTRODUCTION}

In [6], B. Weiss asked for distinguishing invariants for non-dissipative, aperiodic Borel automorphims - especially those which have no finite invariant measures. The purpose of this note is to suggest weakly wandering and some related sequences as invariants for such transformations. We illustrate this with an example of two nonisomorphic Borel automorphisms which have no finite invariant measures. We then show how to construct an uncountable family of non-isomorphic automorphisms.

\section{EXAMPLE}

It is useful for the reader to be familiar with the concepts and notation of [6] (and also [5]). Let $T$ denote a non-dissipative, aperiodic Borel automorphism defined on a standard Borel space $(X, \mathcal{B})$. A set $W \in \mathcal{B}$ is a wandering set for $\mathrm{T}$ if $W \cap T^{n} W=\emptyset$ for $n \neq 0$. Statements and sets are meant to be taken mod the $\sigma$-ideal generated by the wandering sets (or mod the sets of measure zero if one has a favorite quasiinvariant measure in mind). We denote by $\mathcal{W}(X, T)$ the $\sigma$-ideal generated by the wandering sets of $T$.

We begin with an example of two non-isomorphic Borel maps neither of which has a finite invariant measure. With a different representation, this originally appeared in [1] as an example of non-similar infinite measure preserving ergodic transformations of finite type.

We denote by $\mathbb{N}$ the set of all non-negative integers $n \geq 0$, and by $\mathbb{Z}$ the set of all integers. Let $X=\{0,1\}^{\mathbb{N}}$ and define in $X$ the Borel field of subsets generated by the finite rectangles in $X$. For a point $x=\left(x_{0}, x_{1}, \ldots\right) \in X$ we shall say that $x_{i}$ is the $i$-th coordinate of the point $x$. Next we let $T$ be the usual dyadic adding machine on $X$. Namely, we define the operation + in $X$ to be addition $(\bmod 2)$ in each coordinate together with the appropriate carry, and for $x \in X$ define $T x=x+e$ where $e=(1,0,0, \ldots)$.

Received by the editors January 15, 1997 and, in revised form, April 23, 1997.

1991 Mathematics Subject Classification. Primary 28D99.

Key words and phrases. Borel automorphisms, ergodic theory.

(C)1998 American Mathematical Society 
We immediately eliminate from $X$ the countable set of points $\bigcup_{k \in \mathbb{N}}\left\{\left(x_{0}, x_{1}, \ldots\right) \in\right.$ $X \mid x_{i}=0$ for all $\left.i \geq k\right\}$ and $\bigcup_{k \in \mathbb{N}}\left\{\left(x_{0}, x_{1}, \ldots\right) \in X \mid x_{i}=1\right.$ for all $\left.i \geq k\right\}$, and denote the resulting space by $X$ again. It follows that $T$ is a Borel automorphism on $X$.

In what follows we shall be working inside this space $X$ with the transformation $\mathrm{T}$ defined on it as described above.

In the space $X$ we define the two subsets $W_{e}$ and $W_{o}$; namely, $W_{e}$ equals the set of all points $x \in X$ which have their even coordinates equal to 0 , and $W_{o}$ equals the set of all points $x \in X$ which have their odd coordinates equal to 0 . Let us denote $X_{e}=\bigcup_{n \in \mathbb{N}} T^{n} W_{e}$ and $X_{o}=\bigcup_{n \in \mathbb{N}} T^{n} W_{o}$. Both these sets are invariant under $T$. Moreover, $T$ is aperiodic, non-dissipative on each and preserves no finite invariant measure. (We remark that it is easy to construct a $\sigma$-finite infinite invariant ergodic product measure for each.)

Proposition 2.1. There is no Borel isomorphism between $\left(X_{e}, T\right)$ and $\left(X_{o}, T\right)$.

It is a simple observation that for any odd integer $n>0$ we have $T^{n} W_{e} \cap W_{e}=\emptyset$ since each point $x \in T^{n} W_{e}$ has a 1 in the 0 -th coordinate. This is easily extendable to the following:

Lemma 2.2. Let $n>0$ be a positive integer. Then either $T^{n} W_{e} \cap W_{e}=\emptyset$ or $T^{n} W_{o} \cap W_{o}=\emptyset$.

Proof. Let $n>0$ be a positive integer, and suppose that the first time a 1 appears in the dyadic representation of $n$ is in the $i$-th coordinate. If $i$ is an even integer, then $T^{n} W_{e} \cap W_{e}=\emptyset$; otherwise $T^{n} W_{o} \cap W_{o}=\emptyset$.

Proof. Proposition 2.1: Assume $\phi$ is an isomorphism between $\left(X_{e}, T\right)$ and $\left(X_{o}, T\right)$. From Lemma 2.2 it follows that $T^{n}\left(\phi W_{e} \cap W_{o}\right) \cap\left(\phi W_{e} \cap W_{o}\right)=\emptyset$ for all $n>0$. This shows that $\phi W_{e} \cap W_{o}$ is a wandering set. By a similar argument we conclude that $\phi T^{i} W_{e} \cap T^{j} W_{o}$ is a wandering set for any $i, j \in \mathbb{N}$. We also have $X_{o}=$ $\bigcup_{i, j \in \mathbb{N}} \phi T^{i} W_{e} \cap T^{j} W_{o}$; which implies the set $X_{o} \in \mathcal{W}\left(X_{o}, T\right)$.

\section{Definitions}

In this section we list a few useful definitions and make some comments on related notions that led us to the construction of the examples that follow.

Let $T$ denote a non-dissipative, aperiodic Borel automorphism defined on a standard Borel space $(X, \mathcal{B})$.

An infinite sequence of integers $\left\{n_{i}\right\}$ is called a weakly wandering sequence for $T$ if there exists a set $W \in \mathcal{B}$ such that $T^{n_{i}} W \cap T^{n_{j}} W=\emptyset$ for all $i \neq j$. The sequence is called exhaustive weakly wandering if in addition $X=\bigcup_{n_{i}} T^{n_{i}} W$ (disj). A set $W \in \mathcal{B}$ is called of full saturation for $T$ if $\bigcup_{i \in \mathbb{Z}} T^{i} W=X$. Weakly wandering sets and sequences were introduced in [4].

The three collections of weakly wandering sequences $\mathcal{W} W(T)$, exhaustive weakly wandering sequences $\mathcal{E} W W(T)$, and full saturation weakly wandering sequences $\mathcal{F} S W W(T)$ are isomorphism invariants.

From the definitions we have $\mathcal{E} W W(T) \subset \mathcal{F} S W W(T) \subset \mathcal{W} W(T)$. The example in the previous section is a case where the collections $\mathcal{E} W W\left(X_{e}, T\right)$ and $\mathcal{E} W W\left(X_{o}, T\right)$ are not the same; we prove this at the end of the paper.

Observe that if $T$ admits a weakly wandering sequence of full saturation, then there does not exist any finite invariant measure. However the converse is false. 
In [3] an example is given of a transformation with no finite invariant measure where $\mathcal{F} S W W(T)$ is empty. Thus the example in [3] is non-isomorphic to both the examples in the previous section. It will also be non-isomorphic to the examples in the next section.

In order to clarify our arguments and view our examples in a more general setting, it is helpful to consider concepts from the p-adic integers. For example, the space $X$ mentioned above, where all these examples take place, may be regarded as the space of 2-adic numbers with the set of integers removed from it. We define the following object which has a connection with our presentation (this will be demonstrated in the final section of the paper). For an integer $n \in \mathbb{Z}$ let $\operatorname{ord}_{2}(n)$ be the smallest integer $i \in \mathbb{N}$ such that $2^{i}$ divides $n$. In other words, $n=2^{\operatorname{ord}_{2}(n)} m$, where $m$ is an odd integer. We note that $\operatorname{ord}_{2}(n)=0$ if $n$ is an odd integer. We also assume that $\operatorname{ord}_{2}(0)=\infty$.

For a set of positive integers $\mathbb{K}$ let us define $\operatorname{IP}\{\mathbb{K}\}$ to be the set of all integers that are sums of finite subsets of $\mathbb{K}$. In this note we shall assume that 0 corresponds to the empty sum and belongs to every IP set.

It is interesting to observe that in our examples there is a connection among IP sets, weakly wandering sequences, and the $\operatorname{ord}_{2}$ of an integer. For instance, let $\mathbb{S} \subset \mathbb{N}$ be a sequence of non-negative integers. Then for any two integers $n_{1}, n_{2} \in \operatorname{IP}\left\{2^{i} \mid i \in \mathbb{S}\right\}, n_{1} \neq n_{2}$, we have $\operatorname{ord}_{2}\left(n_{1}-n_{2}\right) \in \mathbb{S}$.

\section{An uncountable family}

In this section we construct an uncountable family of non-isomorphic maps. We shall work with the Borel automorphism $T$ defined on the space $X$ that was introduced earlier.

Before we present the main results of this section, we make a few observations that have an interest of their own.

Let us consider an infinite set of non-negative integers $\mathbb{S} \subset \mathbb{N}$ such that $\mathbb{S}^{\prime}$, the complement of $\mathbb{S}$ in $\mathbb{N}$, is also an infinite subset of $\mathbb{N}$. Let $W_{\mathbb{S}}=\left\{x \in X \mid x_{i}=0\right.$ for all $\left.i \in \mathbb{S}^{\prime}\right\}$, and put $X_{\mathbb{S}}=\bigcup_{i \in \mathbb{Z}} T^{i} W_{\mathbb{S}}$. The set $X_{\mathbb{S}}$ then is invariant under the Borel automorphism $T$ defined on $X$. Furthermore, since we initially eliminated from the space the countable set of points mentioned earlier, which consists of the point $0=(0,0,0, \cdots)$ and its orbit under $T$, it follows that $X_{\mathbb{S}}=\bigcup_{i \in \mathbb{N}} T^{i} W_{\mathbb{S}}$ exactly (though, one only needs this modulo the sigma-ideal of wandering sets). Let us also define the set of integers $\mathbb{B}_{\mathbb{S}}=\operatorname{IP}\left\{2^{i} \mid i \in \mathbb{S}^{\prime}\right\}$.

Proposition 4.1. For the Borel automorphism $T$ defined on $X_{\mathbb{S}}$ the set $W_{\mathbb{S}}$ is an exhaustive weakly wandering set under the sequence $\mathbb{B}_{\mathbb{S}}$.

Proof. We note that the set $\mathbb{B}_{\mathbb{S}}$ consists of 0 and all positive integers $n>0$ with the property that for some finite subset $\mathbb{F} \subset \mathbb{S}^{\prime}$ the dyadic representation of $n$ has a 1 in its $i$-th coordinate for $i \in \mathbb{F}$, and a 0 elsewhere. Also the set $W_{\mathbb{S}}$ consists of all points $x \in X_{\mathbb{S}}$ with the property that $x$ has a 0 in its $i$-th coordinate for all $i \in \mathbb{S}^{\prime}$. From this it follows that $X_{\mathbb{S}}=\bigcup_{b \in \mathbb{B}_{\mathbb{S}}} T^{b} W_{\mathbb{S}}(d i s j)$.

Next we observe that it is possible to construct an uncountable family of infinite subsets of positive integers such that any two of them have finite intersection. To show this, consider a 1-1 correspondence between the positive integers and the rationals. For each real number take a sequence of rationals that converge to it. Under the 1-1 correspondence, we obtain the desired family. 
Let $\left\{\mathbb{S}_{\alpha}\right\}$ be an uncountable family of infinite subsets of $\mathbb{N}$ with pairwise finite intersections. We note that for every $\mathbb{S}_{\alpha}$ in this family, $\mathbb{S}^{\prime}{ }_{\alpha}$, the complement of $\mathbb{S}_{\alpha}$ in $\mathbb{N}$, is again an infinite subset of $\mathbb{N}$.

Now let us fix $\alpha \neq \beta$, and let $\mathbb{F}=\mathbb{S}_{\alpha} \cap \mathbb{S}_{\beta}$ denote the finite intersection of the sets $\mathbb{S}_{\alpha}$ and $\mathbb{S}_{\beta}$.

Let $W_{\alpha}=W_{\mathbb{S}_{\alpha}}=\left\{x \in X \mid x_{i}=0\right.$ for all $\left.i \in \mathbb{S}^{\prime}{ }_{\alpha}\right\}$ and $W_{\beta}=W_{\mathbb{S}_{\beta}}=\{x \in$ $X \mid x_{i}=0$ for all $\left.i \in \mathbb{S}_{\beta}^{\prime}\right\}$. Also let $V_{\alpha, \mathbb{F}}=\left\{x \in W_{\alpha} \mid x_{i}=0\right.$ for all $\left.i \in \mathbb{F}\right\}$ and $V_{\beta, \mathbb{F}}=\left\{x \in W_{\beta} \mid x_{i}=0\right.$ for all $\left.i \in \mathbb{F}\right\}$.

We let $X_{\alpha}=\bigcup_{i \in \mathbb{N}} T^{i} W_{\alpha}$, and $X_{\beta}=\bigcup_{i \in \mathbb{N}} T^{i} W_{\beta}$ as before; both of these then are invariant sets under $T$. It is also easy to see that $X_{\alpha}=\bigcup_{i \in \mathbb{N}} T^{i} V_{\alpha, \mathbb{F}}$ and $X_{\beta}=\bigcup_{i \in \mathbb{N}} T^{i} V_{\beta, \mathbb{F}}$.

Theorem 4.2. There is no Borel isomorphism between $\left(X_{\alpha}, T\right)$ and $\left(X_{\beta}, T\right)$.

Using Lemma 4.3 (which replaces Lemma 2.2) that follows, and after replacing the $W$ sets by the corresponding $V$ sets and making the appropriate subscript changes, the proof of Theorem 4.2 follows word for word the proof of Proposition 2.1 above. We omit the details.

Lemma 4.3. For each $n \in \mathbb{N}$ either $T^{n} V_{\alpha, \mathbb{F}} \cap V_{\alpha, \mathbb{F}}=\emptyset$ or $T^{n} V_{\beta, \mathbb{F}} \cap V_{\beta, \mathbb{F}}=\emptyset$.

Proof. We note that if $\operatorname{ord}_{2}(n) \in \mathbb{S}_{\alpha}^{\prime} \cup \mathbb{F}$ then $T^{n} V_{\alpha, \mathbb{F}} \cap V_{\alpha, \mathbb{F}}=\emptyset$. Similarly, if $\operatorname{ord}_{2}(n) \in \mathbb{S}_{\beta}^{\prime} \cup \mathbb{F}$ then $T^{n} V_{\beta, \mathbb{F}} \cap V_{\beta, \mathbb{F}}=\emptyset$. We also have $\left(\mathbb{S}^{\prime}{ }_{\alpha} \cup \mathbb{F}\right) \cup\left(\mathbb{S}_{\beta}^{\prime} \cup \mathbb{F}\right)=\mathbb{N}$.

It is interesting to note that if one looks to the proof of Proposition 2.1, it is possible to conclude a more general theorem.

Let us consider two non-dissipative and aperiodic automorphisms $S$ and $T$ defined on the standard Borel spaces $(X, \mathcal{B})$ and $(Y, \mathcal{F})$, respectively. Let $E \subset X$ be a set of full saturation for $S$; that is $X=\bigcup_{i \in \mathbb{Z}} S^{i} E$, and define $\mathcal{R}(E, S)=$ $\left\{n \in \mathbb{Z} \mid S^{n} E \cap E \neq \emptyset\right\}$. Similarly, for a set $F \subset Y$ of full saturation for $T$ define $\mathcal{R}(F, T)=\left\{n \in \mathbb{Z} \mid T^{n} F \cap F \neq \emptyset\right\}$. Let us denote by $\mathcal{R}^{\prime}(E, S)$ and $\mathcal{R}^{\prime}(F, T)$ the complements in $\mathbb{Z}$ of the sequences $\mathcal{R}(E, S)$ and $\mathcal{R}(F, T)$, respectively.

We state Theorem 4.4 below as a generalization to Theorem 4.2. We omit the proof.

Theorem 4.4. Let $S$ and $T$ be two non-dissipative, aperiodic Borel automorphisms defined on $X$ and $Y$, respectively, and let $E$ and $F$ be two sets of full saturation for $S$ and $T$, respectively. Then $\mathcal{R}^{\prime}(E, S) \cup \mathcal{R}^{\prime}(F, T)=\mathbb{Z}$ implies $(X, S)$ is not Borel isomorphic to $(Y, T)$.

\section{EXAMPLE REDUX}

In this section, we prove the following, which was stated earlier. The proof illustrates how the various concepts presented in the paper are interrelated.

Proposition 5.1. $\mathcal{E} \mathcal{W W}\left(X_{e}, T\right) \neq \mathcal{E} \mathcal{W W}\left(X_{o}, T\right)$.

We already have that $\mathbb{B}=\operatorname{IP}\left(2^{2 n}: n \geq 0\right\} \in \mathcal{E} \mathcal{W} \mathcal{W}\left(X_{e}, T\right)$. So, it is enough to show that $\mathbb{B} \notin \mathcal{E} \mathcal{W} \mathcal{W}\left(X_{o}, T\right)$. We also have $\operatorname{IP}\left(2^{2 n+1}: n \geq 0\right\} \in \mathcal{E} \mathcal{W} \mathcal{W}\left(X_{o}, T\right)$ and in particular $W_{o} \cap T^{n} W_{o} \emptyset$ for all $n>0$ of odd $\operatorname{ord}_{2}$.

Let $W$ be an exhaustive weakly wandering set under the sequence $\mathbb{B}$ for $\left(X_{o}, T\right)$. The proof of the proposition follows, using the lemma below, in a similar manner to the non-isomorphism result above. 
Lemma 5.2. $W \cap T^{n} W=\emptyset$ for all $n$ of even $\operatorname{ord}_{2}$.

Proof. Let $\operatorname{ord}_{2}(n)=2 k$ and let $\mathbb{B}_{k}=\operatorname{IP}\left(2^{2 i}: i \geq 0, i \neq k\right\}$. We denote by $X_{k}=$ $\bigcup_{b \in \mathbb{B}_{k}} T^{b} W$. Then $X_{o}=X_{k} \cup T^{2^{2 k}} X_{k}\left(\right.$ disj), and applying $T^{2^{2 k}}$ to $X_{o}$ we conclude that $X_{o}=T^{2^{2 k}} X_{k} \cup T^{2^{2 k+1}} X_{k}$ (disj). This implies $T^{2^{2 k+1}}\left(T^{2^{2 k}} X_{k}\right)=T^{2^{2 k}} X_{k}$. Since $\operatorname{ord}_{2}(n)=2 k$, we have $n=2^{2 k}+q 2^{2 k+1}$ for some $q \geq 0$. This shows that $T^{n} W \in T^{2^{2 k}} X_{k}$ and hence is disjoint from $W$.

\section{REFERENCES}

1. S. Eigen, A. Hajian and Y. Ito, Ergodic measure preserving transformations of finite type, Tokyo J. of Math 110, (1988) 459-470. MR 90b:28017

2. S. Eigen, A. Hajian and S. Kakutani, Complementing sets of integers - a result from ergodic theory. Japanese J. Math. 18, No. 1, (1992) 205-211. MR 93g:11087

3. S. Eigen, A. Hajian and M.G. Nadkarni, Weakly wandering sets and compressibility in descriptive setting, Proc. Indian Acad. Sci. 103, (1993) 321-327. MR 95g:28028

4. A. Hajian and S. Kakutani, Weakly wandering sets and invariant measures, Trans. Amer. Math. Soc. 110 (1964) 136-151. MR 27:4904

5. M.G. Nadkarni, Descriptive ergodic theory, in Contemporary Mathematics 94, American Math. Society, (1989) 191-206. MR 90h:28021

6. B. Weiss, Measurable dynamics in Contemporary Mathematics Volume 26, American Math. Society, (1984) 395-421. MR 85j:28027

(S. Eigen and A. Hajian) Department of Mathematics, Northeastern University, Boston, MASSACHuSETTS 02115

E-mail address: eigen@neu.edu

E-mail address: hajian@neu.edu

(B.Weiss) Department of Mathematics, The Hebrew University of Jerusalem, Institute of Mathematics, Givat Ram 91904 Jerusalem, Israel

E-mail address: weiss@math.huji.ac.il 